\title{
Entre étudiants sur le tard et apprentis-professionnels
}

\author{
Figures de la professionnalisation des doctorants \\ en sciences humaines et sociales
}

Clémentine Gozlan ${ }^{1}$

\begin{abstract}
[Résumé] Cet article montre que le développement de nouveaux dispositifs pour favoriser l'intégration professionnelle des doctorants s'inscrit dans des conditions politiques, institutionnelles et scientifiques. Nous abordons l'évolution du rôle et de la place du doctorant « de biais », telle qu'elle se donne à lire dans les discours et les réformes menées par les acteurs qui orientent les politiques scientifiques depuis les années 1990 en France. La diversité des situations des doctorants en sciences humaines et sociales en France nous invite à appréhender leurs conditions de travail et leurs orientations professionnelles comme tributaires de facteurs sociaux - tels que l'ouverture de leur discipline au marché et à la société civile, le degré de division du travail et les modes de production du savoir dominant, dont la forme et l'importance varient selon les disciplines. Le sort hétérogène des doctorants peut alors être considéré comme un révélateur de l'organisation du travail scientifique en France.

Mots-clés: Doctorants, professionnalisation, politiques scientifiques, sciences humaines et sociales, disciplines.
\end{abstract}

[Abstract] This article evidences that the development of new devices favoring PhD students' professional integration are aligned with political, institutional and scientific conditions. I analyze the evolution of young researchers' situation from above, as one can derive it from the reforms and discourses held by actors who orientate France's scientific policies since the 1990s. The diversity of doctoral students' situations in the humanities and social sciences invites to apprehend their working condition and their professional orientations as depending on social factors - such as the openness of their discipline to the market and civil society, the degree of labor division and the dominant modes of knowledge production, which vary across disciplines. Thus, France's doctoral students' heterogeneity reveals how the scientific work is organized in France.

Keywords: Doctoral Students, Professionalization, Scientific Policies, Humanities and Social Sciences, Disciplines.

\footnotetext{
${ }^{1}$ Centre de sociologie des organisations, CNRS, Sciences Po, Paris.
} 


\section{Introduction}

Janvier 2014. Comité d'évaluation AERES d'un laboratoire de recherche en littérature.

Banlieue parisienne. Rencontre à huis-clos entre les évaluateurs et les doctorants².

$11 \mathrm{~h} 30$ : Le président du comité d'évaluation s'adresse aux doctorants du laboratoire : «Est-ce que vous êtes heureux ? Dans cet environnement doctoral, ça a l'air sur le papier, mais est-ce que vous êtes vraiment au sein d'une équipe ? Parlez librement, tout ce qui est dit l'est hors micro [...]. Si vous avez des doléances ou des problèmes, c'est le moment !».

Au-delà de son caractère anecdotique, ce verbatim tiré d'une observation réalisée en 2014 lors d'un comité d'évaluation d'une équipe de recherche en littérature illustre l'attention que portent les organes chargés d'évaluer la qualité des centres de recherche, tels qu'en France, l'Agence d'évaluation de la recherche et de l'enseignement supérieur (AERES'), à l'égard des doctorants et jeunes chercheurs. L'institution d'une rencontre entre les doctorants et les experts lors de l'évaluation des laboratoires de recherche signale d'une part qu'ils se voient accorder une place formalisée au sein des structures collectives de recherche, et ce, quelle que soit la discipline. D’autre part, comme le suggère l'adresse liminaire retranscrite ci-dessus, le bien-être des doctorants (" êtes-vous heureux » ?), leur intégration dans le laboratoire (" est-ce que vous êtes vraiment au sein d'une équipe ?»), font partie des préoccupations dont les membres permanents des centres de recherche doivent avoir le souci, s'ils souhaitent obtenir une évaluation favorable.

L'attention à l'égard du sort des doctorants n'est pas récente. En France, les écoles doctorales ont été créées dans les années 1990 pour assurer et centraliser le suivi des doctorants. Leur rôle et leurs moyens ont longtemps été faibles, mais leur création illustre la considération des pouvoirs publics envers les jeunes chercheurs. À l'échelle

\footnotetext{
${ }^{2}$ L'utilisation du genre masculin a été adoptée à la seule fin d'alléger le texte.

${ }^{3}$ Cette agence a été créée en 2007. Elle était chargée d'organiser l'évaluation de l'ensemble du dispositif français de recherche et d'enseignement supérieur, et de construire des méthodes d'évaluation unifiées et transversales à toutes les disciplines académiques. Chaque structure est évaluée tous les quatre ans (tous les cinq ans, depuis 2011). L'évaluation des laboratoires donne lieu à une note rendue publique (A+, A, B ou C). Suite aux controverses suscitées par la mise en place de cette agence, un Haut conseil de l'évaluation de la recherche et de l'enseignement supérieur (HCERES) la remplace depuis 2014. Ses attributions restent proches.
} 
européenne, la réforme de l'architecture des diplômes et l'accent placé sur l'enseignement supérieur et sur la recherche comme vecteur de la compétitivité des nations montrent que la place et la formation des doctorants sont devenues des enjeux phares de ce secteur. Les jeunes chercheurs s'autosaisissent aussi du discours sur leur propre parcours professionnel. En témoigne le foisonnement de blogs, de témoignages, d'ouvrages (La prise de thèse, d'Elsa Gisquet, publié en 2013), de bandes dessinées (Carnet de thèses, de Tiphaine Rivière, paru en 2015), qui mettent en visibilité ce que nous pourrions appeler la « condition doctorale » et/ou prodiguent des « guides » à l'attention du jeune chercheur. Enfin, la forte mobilisation de collectifs de doctorants contre la proposition de créer, dans certaines grandes écoles et établissements universitaires français, un doctorat alternatif, d'une durée d'un an ou de deux, à destination de salariés du privé qui paieraient d'importants frais d'inscription ${ }^{4}$, montre enfin que les jeunes chercheurs s'investissent dans la défense de leurs compétences : ils réaffirment la valeur de leur diplôme, qui sanctionne une expertise et des savoir-faire acquis sur un plus long terme, dans un univers scientifique.

Ces évolutions permettent de questionner le processus de professionnalisation des doctorants, entendue selon deux dimensions complémentaires. Celle d'abord de leur reconnaissance sociale en tant que groupe qui dispose d'une place spécifique dans la division du travail. Cette reconnaissance émane tant du « haut », de la puissance publique et des institutions universitaires, que du « bas » ou de l'intérieur ; les jeunes chercheurs émettant des revendications relatives à leur statut social et à la valeur de leur formation, et ce faisant, se différencient - s'autonomisent - d'autres groupes susceptibles de détenir des compétences savantes. La professionnalisation s'entend donc comme le processus, parfois inachevé ou problématique (Demaziere, 2009), de reconnaissance sociale d'un groupe en tant que tel. Mais elle repose également sur l'apprentissage de codes, de normes et de savoirs, que les individus incorporent au cours de leur socialisation au sein d'un univers professionnel donné.

Cet article montre que ces deux dimensions de la professionnalisation peuvent être en tension. En effet, l'attention de la puissance publique et des jeunes chercheurs euxmêmes à leur professionnalisation s'inscrit paradoxalement dans un contexte d'incertitude relative aux débouchés et à l'employabilité des jeunes docteurs, principalement en sciences humaines et sociales 5 . Leur professionnalisation est équivoque : si l'accent est placé sur la formation et l'acquisition de compétences des jeunes chercheurs, d'une

\footnotetext{
${ }^{4}$ Voir notamment l'article suivant: http://rue89.nouvelobs.com/2015/02/23/sciences-podoctorat-professionnel-vente-diplomes-encheres-257865.

${ }^{5}$ Pour une synthèse relative à l'insertion professionnelle des docteurs, voir : http://www.letudiant.fr/static/uploads/mediatheque/EDU_EDU/0/1/138601-les-difficultes-d-insertion-professionnelle-des-docteurs-mohamed-harfi-cgsp-octobre-2013-original.pdf.
} 
part, ces professionnels en devenir se situent à la lisière de plusieurs marchés du travail. Cette situation rompt avec le modèle anglo-saxon des professions établies (Freidson, 1984) où, une fois passée la barrière de l'entrée dans la profession - ici le doctorat le jeune impétrant appartient à une profession donnée. D'autre part, le fait professionnel recouvre des réalités distinctes : les modalités de la professionnalisation des doctorants, leur place et leur forme de socialisation au sein des collectifs de recherche ${ }^{6}$ sont variables.

Il apparaît alors d'autant plus fécond d'analyser conjointement le rôle d'instances régulatrices de la profession dans les évolutions de la condition doctorale, qui promeuvent un ensemble de dispositifs permettant de construire l'employabilité des doctorants, ainsi que les conditions de l'effectivité de ces injonctions. Prolongeant les travaux contemporains qui ont battu en brèche une vision unifiée des groupes professionnels (Demazière et al., 2009), nous saisissons cependant l'hétérogénéité fondamentale du professionnalisme à un moment clé où il se définit, à savoir au moment de l'entrée dans le métier. En proposant un cadre d'analyse des formes hétéroclites de la socialisation professionnelle, c'est plus largement sur le fonctionnement et l'organisation du monde scientifique que renseigne cet article.

Pour ce faire, nous nous centrons sur les doctorants en sciences humaines et sociales. D'une part, ils constituent une population qui n'a été que peu étudiée par les travaux de sciences sociales, à de notables exceptions, telles que le numéro spécial consacré à la socialisation professionnelle des doctorants en sciences sociales (Serre, 2015). En effet, les travaux portent plus volontiers sur les jeunes chercheurs en biologie et chimie (Louvel, 2006, Frances, 2012), en nanotechnologie (Barrier, 2011), en physique (Shinn, 1998). Prendre pour objet des domaines scientifiques dans lesquels la division technique du travail est généralement moins marquée ${ }^{7}$ permettait de compléter ces approches.

Les données utilisées dans cet article sont tirées de l'enquête réalisée dans le cadre de notre thèse de sociologie sur l'évaluation de la qualité de la recherche en Sciences Humaines et Sociales (SHS). Les méthodes sont principalement qualitatives : sont mobilisés des entretiens avec des directeurs de laboratoire, des membres de l'AERES et du

\footnotetext{
${ }^{6}$ Nous replaçons les doctorants dans leur environnement de travail institutionnel, à savoir en France, les laboratoires de recherche, ce pourquoi nous ne discuterons pas ici la professionnalisation sous l'angle des pratiques d'enseignement, dont nous soulignons ici qu'elles sont déterminantes dans le cadre la qualification par le Conseil National des Universités (CNU).

${ }^{7}$ L'article de Shinn montre bien, dans le cas de la physique, que les tâches et les types de travaux conduits dans les laboratoires sont différenciés en fonction du statut des membres qui le composent. En sciences humaines et sociales, la spécialisation des travaux en fonction du statut au sein des centres de recherche n'est généralement pas aussi manifeste. Des enquêtes montrent aussi notamment que les lieux et les conditions de travail des doctorants varient fortement selon qu'ils appartiennent aux « sciences » ou aux sciences humaines et sociales (Vilter, 2008).
} 
ministère de la Recherche, et une partie de la littérature grise produite par ces institutions.

Encadré : la position duale des enquêtés

Les membres de l'AERES sont presque exclusivement des enseignants-chercheurs et des chercheurs. Il en est de même pour les scientifiques du ministère de la recherche que nous citons dans cet article. Ces professeurs continuent d'enseigner et de produire de la recherche, mais ils se distinguent par le fait qu'ils ont pour la plupart occupé des fonctions dans l'administration de la recherche (par exemple, en ayant dirigé un ou plusieurs laboratoires, en ayant été doyen de Faculté, Vice-Président Recherche à l'Université, etc.). Ils sont nombreux à s'être investis dans l'évaluation scientifique ou institutionnelle, à différentes échelles. Cette position spécifique leur fournit une double ressource pour légitimer les réformes qu'ils encouragent : d'une part, celles-ci n'émanent pas de « managers » externes à la profession, ces acteurs revendiquant leur appartenance à la sphère scientifique. D'autre part, situés sensiblement « au-dessus » de la base professionnelle en ayant circulé dans des arènes décisionnelles, ils peuvent faire valoir leur connaissance des institutions académiques pour promouvoir leur vision de la formation légitime au métier d'enseignant-chercheur.

L'étude de ces sources est complétée par une analyse quantitative, réalisée à partir d'un échantillon de rapports d'évaluation de l'AERES.

Nous retraçons d'abord l'émergence progressive des injonctions réformatrices que des décideurs ou les membres d'instances régulatrices de la profession font peser sur les centres de recherche en faveur des doctorants en SHS. Cependant, derrière cette convergence apparente dans les discours, les types d'activités des doctorants, leur place dans les collectifs de recherche, sont hétérogènes. Cette diversité des configurations nous invite à appréhender les conditions de travail et de formation des doctorants comme tributaires de facteurs sociaux - tels que les modes de production du savoir dominants, la place du marché dans la vie académique - dont la forme et l'importance varient selon les disciplines. Cette étude révèle l'hétérogénéité des modes de professionnalisation au sein d'un monde social donné. 


\section{1. Émergences et formes de l'attention publique aux doctorants en SHS}

\subsection{Le souci des doctorants: des discours anciens aux réformes contemporaines}

Depuis la mise en place des écoles doctorales en France en 1992, le doctorant - ce personnel temporaire des centres de recherche, entre deux âges professionnels, à la fois étudiant et travailleur (Louvel, 2006) - a pris une place de plus en plus conséquente dans les discours des autorités publiques relatives au monde académique. Lorsque le Ministère de la recherche habilitait une équipe de recherche, le formulaire d'évaluation comportait dès les années $1990^{8}$ des rubriques concernant les doctorants : leur nombre tout d'abord, et le ratio entre le nombre d'inscrits et le nombre de thèses effectivement soutenues. Ces premières formes d'évaluation de la formation doctorale, sous forme quantitative, ne sont pas à négliger. Comme le soulignent les travaux de W. Espeland (2015), la quantification peut constituer une étape essentielle dans la mise en visibilité et la constitution d'un phénomène social. Cette rubrique s'est progressivement étoffée, pour s'ouvrir à des considérations plus qualitatives. Au début des années 2000, il est ainsi demandé aux évaluateurs de commenter la qualité de l'encadrement des doctorants, la qualité des thèses soutenues, lorsqu'ils émettent leur recommandation sur la création, le maintien ou la suppression d'un centre de recherche. La progressive prise en compte du traitement et de la place des doctorants dans l'expertise des laboratoires s'est effectuée sous l'impulsion d'un groupe de réformateurs en position influente au ministère de la recherche à cette période, comme le souligne une enquêtée :

En 1998, le discours d'Allègre [ministre de la recherche] était fascinant parce qu'il [...] configurait sa vision de l'université autour de l'étudiant, ce qui était original, notamment autour des doctorants. [...]. II avait une autre vision des doctorants que celle qui se pratiquait dans les universités à l'époque. II y avait d'ailleurs au cabinet d'Allègre deux jeunes docteurs qui incarnaient cela. (Directrice scientifique au Ministère, 1998-2002, SHS)

Cependant, les enquêtés ayant occupé des fonctions décisionnelles au tournant des années 2000 sont les premiers à reconnaître que ces efforts envers une meilleure prise en compte des besoins des doctorants était davantage de l'ordre du discours que des pratiques :

Nous savions très bien que la Sorbonne et les grandes universités parisiennes, les gens acceptent soixante doctorants, on ne suit pas soixante doctorants, ça c'est vraiment une évidence. [...] Mais on n'évalue pas négativement un professeur à la Sorbonne. Sinon on le paye plus tard. (Coordinateur scientifique, ministère, SHS)

\footnotetext{
${ }^{8}$ Sources : Archives Nationales (France), versement 20080487, art. 1 à 3.
} 
Les enquêtés mettent ainsi en avant la lente mise à l'agenda public de la nécessité d'encadrer et d'accompagner les doctorants, tout en soulignant que les moyens de pression pour la rendre effective étaient limités.

Les formes de l'attention aux doctorants changent en 2007. Avec la mise en place de l'AERES, nous assistons à une formalisation sans précédent des missions des centres de recherche. Parmi celles-ci, l'implication des titulaires dans le suivi des doctorants (principalement pendant la thèse, mais aussi après) occupe une place croissante. Ainsi, en 2012, un critère d'évaluation dédié à cette dimension de l'activité des laboratoires a vu le jour suite à une consultation de plusieurs mois. Dans le document interne que nous reproduisons ci-dessous, nous pouvons voir l'échelle de valeur promue par les membres de l'AERES :

« Implication dans la formation par / à la recherche :

Note A+ : Intégration structurée des étudiants dans les activités scientifiques de l'unité, du master au doctorat. Accompagnement des étudiants dans le cadre de leur expérience professionnelle. Offre de cours et de séminaires de recherche riche et diversifiée, de renommée internationale, au niveau doctoral. Mise en œuvre d'une stratégie de suivi des docteurs. [...].

Note C : Déficit sur plusieurs des indicateurs (par exemple, absence de suivi des doctorants et des docteurs; absence de formation proposée aux étudiants de masters et aux doctorants ; participation inexistante des doctorants à la vie de l'entité $)^{9}$. »

Avec l'AERES, les doctorants sont considérés comme des membres, certes temporaires, mais à part entière des laboratoires, quelle que soit la discipline considérée. Les incitations à les intégrer dans le laboratoire et à mettre en place un suivi doctoral sont instituées dans les documents que doivent fournir les laboratoires à l'AERES. S'instaure alors une véritable bureaucratie du contrôle de l'encadrement doctoral, qui contraint les responsables de centre à tenir un tableau de bord détaillé. Une partie de la profession académique en SHS déplore la lourdeur administrative et la procéduralisation du suivi doctoral, estimant que les indicateurs requis sont impossibles à renseigner :

Mais c'est trop lourd! Dans les documents, il y a un formulaire qui demande toutes les dates de naissance de tous les doctorants. C'est de la folie parce que les universités n'ont pas ces données là. Les directeurs de centres font souvent le secrétariat tous seuls, et par exemple, je n'avais pas mis les dates de naissance des doctorants [...], je ne me suis pas embêté avec ça, et on me l'a reproché. Je

\footnotetext{
${ }_{9}^{9}$ Archives personnelles, Directeur B de la section d'évaluation des laboratoires, AERES, PowerPoint « Pour de nouveaux critères d'évaluation », 12 janvier 2012.
} 
trouve ça bureaucratique. (Directeur de laboratoire de recherche, littérature, au sujet de l'évaluation de son centre par l'AERES)

Dans la mesure où l'évaluation s'achève par l'attribution de notes aux laboratoires, consultables sur internet, chacun des critères de l'AERES devient un enjeu important pour les responsables des centres de recherche, qui s'évertuent à y répondre en transformant le cas échéant leurs pratiques internes, s'ils souhaitent éviter une stigmatisation publique. Ainsi, le traitement des doctorants et leur positionnement dans le centre sont devenus des indices du bon fonctionnement des collectifs de recherche. Témoin de leur attractivité (lorsqu'elles parviennent à recruter des doctorants hors de leur université de rattachement), ou encore de leur vitalité et de leur dynamisme (lorsque l'équipe les intègre à des travaux collectifs), force est de constater que le sort réservé aux jeunes devient l'un des prismes par lequel se mesure la qualité d'un laboratoire de recherche toutes les disciplines confondues.

La dimension performative de l'affichage de critères « de qualité » est assumée, voire revendiquée par les responsables de l'AERES. L'acculturation des collectifs de recherche à des pratiques « vertueuses », au moyen de techniques rappelant celles du benchmarking, est l'un des effets escomptés de l'AERES, selon plusieurs de ses dirigeants :

J'ai entendu un professeur d'université, qui dit : "un professeur d'université à la totale liberté de sa recherche". Moi je traduis ça par : "il a le droit d'avoir autant de thésards qu'il veut, de les massacrer en les accompagnant." [...]. J'ai créé les indicateurs qui ont été publiés, avec la durée des thèses, la durée moyenne, la durée la plus longue, le nombre de thésards moyens par encadrant et le nombre record, [...] il y avait un prof qui encadrait cent-vingt doctorants en droit. C'est aussi un des avantages de l'AERES, c'est qu'en publiant tout cela, ça a quand même fait évoluer le milieu, même en droit. (Directeur $C$ de la section des évaluations des formations et des diplômes, AERES, Professeur des Universités, Sciences expérimentales)

Les premiers décideurs institutionnels de l'AERES s'accordent à défendre la nécessité de réformer les conditions de suivi des doctorants accueillis dans les laboratoires pour se former à la recherche, de sorte à valoriser les «bonnes pratiques » telles qu'ils se les représentent et à proscrire les autres. Généralement, les enquêtés prennent appui sur leur propre expérience dans la recherche pour justifier les réformes qu'ils promeuvent. Ainsi, une Directrice scientifique au ministère se souvient de son entrée dans le monde académique :

«À mon très vénéré maître [...] je faisais dans le meilleur des cas une visite [par] an. [...]. Le bistrot du coin était le lieu de rencontre des doctorants, on minutait combien de temps chacun avait passé dans le bureau. 35 minutes était le record. [...]. Moi, j'ai su par expérience ce que c'était que de se débrouiller toute seule en face d'un sujet avec un grand rire encourageant à chaque rencontre. J'ai toujours dit que mon intérêt pour les doctorants était quand même fondé sur le fait que je souhaitais qu'ils ne vivent jamais ce que j'avais vécu. » 
Forts de leur mémoire des conditions d'existence des thésards dans les années 1980, ils relatent qu'ils ont contribué à instiller, à leur échelle, la prise de conscience de la nécessité de prêter attention au vécu des jeunes chercheurs. Certains d'entre eux placent alors la focale sur un contrat moral qui lie le doctorant et son directeur. Ces injonctions visent à transformer le rôle des directeurs : ils sont appelés à devenir de véritables formateurs dans un contexte d'apprentissage professionnel, plutôt que des professeurs suivant de loin leurs étudiants. Cette évolution des rapports entre doctorants et directeurs fonctionne comme des reflets discrets de l'attention à la professionnalisation des doctorants, dans la mesure où elle révèle l'attention portée à la socialisation des jeunes dans un environnement de travail, vecteur d'insertion professionnelle future.

Enfin, ces discours normatifs sur les pratiques d'encadrement doctoral légitimes et sur la professionnalisation des doctorants au travail académique ${ }^{10}$ peuvent d'autant plus être suivis d'effets que les acteurs institutionnels qui les endossent sont en mesure, par les positions qu'ils occupent dans l'administration scientifique, de les diffuser dans leurs propre laboratoire de recherche, voire de les traduire dans des référentiels d'évaluation des laboratoires.

Ainsi, dans un échantillon de soixante rapports d'évaluation des laboratoires de littérature et de géographie, à partir desquels nous avons constitué une base de données ${ }^{11}$, nous observons que les principaux points forts soulignés par les évaluateurs en ce qui concerne les doctorants sont leur « participation aux activités du centre » (1/6 des rapports le mentionnent), tandis que les recommandations convergent sur la nécessité d'un « meilleur encadrement doctoral » (1/6 des rapports) d'une meilleure « intégration des doctorants au sein du laboratoire ou de l'équipe » (1/10e), dont les modalités peuvent être variées. Plusieurs rapports suggèrent la mise en place d'un comité de thèse, d'un séminaire de doctorants, et incitent - cette mention n'apparaît pas dans les rapports en littérature - le laboratoire à aider les doctorants à publier.

\footnotetext{
${ }^{10}$ Même dans la période contemporaine, les enquêtés en SHS ne mentionnent que très discrètement l'attention aux débouchés professionnels non académiques pour les doctorants. Ce point sera analysé infra.

${ }^{11}$ Nous avons choisi de nous centrer sur l'évaluation de disciplines qui, tout en appartenant aux SHS, se caractérisent par des méthodes, des pratiques professionnelles et des positions dans l'espace académique en parties dissemblables (Bourdieu, 1984). Leur degré d'autonomie ou d'hétéronomie est d'abord variable : si le pôle de la littérature à l'université s'est constitué comme relativement autonome des demandes sociales et politiques - bien que la situation ait évolué depuis la période considérée dans ces enquêtes -, certains segments de la géographie ont été davantage aux prises avec des formes d'applications sociales directes. Enfin, les révolutions méthodologiques qu'ont connues ces disciplines n'ont pas été conduites au même rythme ni avec la même ampleur. Tandis que les méthodes de travail en géographie requièrent de plus en plus, selon les enquêtés, des modes collectifs de travail, notamment depuis l'introduction de la modélisation et de l'analyse spatiale dans les années 1970, cette évolution - sans être inexistante - est moins perceptible en lettres.
} 
Mais outre les efforts de plusieurs décideurs institutionnels, le leitmotiv d'un encadrement doctoral soutenu en SHS s'inscrit également à la croisée de réformes des modes d'organisation de la recherche.

\subsection{L'intégration des doctorants, témoin du développement d'une " culture de laboratoire " en SHS ?}

L'attention au développement de leurs savoir-faire professionnels participe d'une reconnaissance des doctorants comme de futurs enseignants-chercheurs (Louvel, 2006), inscrits dans des collectifs de travail. Or, jusque dans les années 1980 en France, la recherche en SHS n'était pas configurée autour d'équipes ou de collectifs de recherche. Par ailleurs, la thèse délivrée était un « Doctorat d'État », réalisé sur une période d'au moins une dizaine d'années pour les SHS. Le travail de recherche était conduit, selon les enquêtés, de façon solitaire et destiné principalement aux métiers académiques. Plusieurs réformes d'ampleur ont contribué à modifier la place et le statut du doctorant. Afin d'accélérer les carrières, notamment en SHS, la décision a été prise de modifier le format du doctorat. La loi Savary 1984 remplace alors le doctorat d'état par un "Doctorat de $3^{e}$ cycle ", réputé moins long. Parallèlement, les règles d'admission des doctorants se formalisent : aujourd'hui, l'apprenti-professionnel doit obligatoirement être rattaché à une équipe ou un laboratoire de recherche pour réaliser son doctorat $\mathrm{t}^{12}$. Comme le résume un professeur des universités en littérature :

Quand j'ai commencé à l'université où il y avait la thèse d'État, il n'y avait pas d'équipe, [c'était] l'anomie, ça voulait dire qu'il n'y avait pas vraiment de régulation, de socialisation, d'intérêt commun et de sens même d'une collectivité pour les enseignants en lettres. Il y a d'abord la tradition française qui est que, quand il y avait la thèse d'État, c'était un travail d'engagement personnel. L'organisation des équipes aujourd'hui, pour moi c'est la suite logique de la disparition de la thèse d'État.

Ainsi, la conjonction de la réforme des modalités d'obtention du doctorat et de la création de collectifs de recherche intégrés éclaire l'évolution de la place et du rôle du doctorant.

Mais c'est surtout à l'AERES que le caractère collectif du travail de recherche en SHS est mis en exergue. Ses premiers dirigeants - des physiciens -, se sont particulièrement investis pour inciter les disciplines des SHS à se structurer autour d'équipes intégrées :

En SHS, il faut lutter contre ces organisations dans lesquelles les étudiants sont pas encadrés, [...] il y avait un sociologue parisien qui encadrait quatre-vingt thèses, ce n'est pas possible. Vous en laissez soixante-dix dans la nature et vous

\footnotetext{
${ }^{12}$ Cette règle vaut également pour les enseignants-chercheurs. Le Ministère de la recherche estimait, en 2000, à environ un tiers la proportion d'enseignants-chercheurs en SHS qui n'appartenaient à aucun centre de recherche.
} 
en faites souffrir dix. Ça, ce n'est pas bien. (Directeur A de la section d'évaluation

des laboratoires, AERES, Professeur des Universités, physique)

Selon une partie des membres de l'AERES, la formation doctorale ne peut être assurée que dans et par le développement d'une « culture de laboratoire », caractérisée par la mise en présence récurrente de ses membres qui travaillent en collaboration, dans un lieu commun, le centre de recherche, et par l'attention soutenue des équipes aux compétences académiques que doivent développer les jeunes chercheurs.

Le postulat implicite de ces prises de positions est celui selon lequel la socialisation est l'une des clés de la formation des doctorants et un vecteur de leur professionnalisation. Les discours récurrents sur la nécessité de l'encadrement doctoral peuvent être lus à la lumière de cette représentation de l'apprentissage d'un métier par interactions régulières et inscription dans un environnement de travail formalisé. La solitude du doctorant, en sciences sociales notamment (voir Chao et al., 2015), est alors le premier mal à curer, en ce qu'elle constitue l'anti-modèle de cette vision du fait professionnel.

Cette tendance à l'évolution du statut du doctorant dans les laboratoires ne doit cependant pas occulter des variétés notables entre les différentes disciplines, que nous proposons à présent d'explorer.

\section{Des évolutions contrastées. Le poids des modes de travail disciplinaires sur les formes de professionnalisation des doctorants}

La mise en application de ce modèle « idéal » d'organisation de la recherche - dans lequel le doctorant est associé à la production de la recherche du laboratoire et dans l'organisation de ses activités, ce par quoi il se professionnalise - apparaît tributaire de plusieurs facteurs sociaux. Nous avons identifié deux axes de tension majeurs sur lesquels les disciplines se positionnent différemment: le premier concerne les perspectives de débouchés des doctorants après l'obtention de leur diplôme, le second est relatif aux modes de production du savoir des disciplines académiques. Ces deux facteurs sont influencés, quoique de façons variables selon les domaines, par les incitations de plus en plus prégnantes des pouvoirs publics en faveur du développement de la recherche sur projets, et par l'accent placé sur l'utilité socio-économique de la science.

\subsection{Du pôle académique au pôle entrepreneurial ?}

En France, plusieurs dispositifs ont été mis en place de sorte à favoriser l'insertion hors du monde académique des doctorants. La création des thèses Cifre par exemple (conventions industrielles de formation par la recherche), en 1981, permet de financer les doctorants et les entreprises qui les accueillent. Le Ministère estimait en 2012 que 93 \% 
des docteurs Cifre trouvent un emploi dans le secteur privé13. D’autres types de mécanismes favorisant les rapprochements entre jeunes chercheurs et entreprises ont été étudiés. Un article de J. Frances (2012) analyse ainsi les « Doctoriales » en biologie, journées rassemblant des professionnels non académiques et des doctorants, comme l'un des principaux témoins de la transformation du doctorant en entrepreneur. Les injonctions des pouvoirs publics en faveur des thèses financées plutôt que non financées ${ }^{14}$, couplée à la diminution des allocations doctorales attribuées par le Ministère de la recherche aux laboratoires, incitent les centres de recherche à se tourner vers des bailleurs de fonds publics ou privés, et indirectement, à répondre à leur commande :

Il faut bien aller à un moment aussi là où i y a le pognon et du coup on est poussé

à rentrer dans certaines cases dans lesquels on n'avait pas forcément prévu de rentrer [...] quand on voit le nombre de recherches qui sont effectivement dans tout ce qui est développement durable c'est lié au fait qu'il y a des mannes de financement pour ça. (Chargée de recherche, sociologie urbaine, CNRS)

Pourtant, nous ne pouvons conclure à l'hégémonie de logiques marchandes sur l'orientation des jeunes chercheurs ${ }^{15}$. D'une part, car la place occupée par le marché est très différente selon les disciplines. En 2004, les SHS n'occupent que $0,2 \%$ des chercheurs en entreprise, contre $30 \%$ en Sciences pour l'ingénieur ${ }^{16}$. Mais à l'intérieur même des SHS, les configurations sont variables. Si en architecture, par exemple, les enquêtés soulignent qu'ils « s'efforcent de former à une profession qui travaille pour le monde réel »(Délégué scientifique AERES, géographie, urbanisme, architecture), en littérature en revanche, les perspectives d'emploi hors du monde académique sont plus rares :

Il y avait un modèle d'étudiants : [...] "il a déjà un contrat avec je ne sais pas quoi".

Moi les pauvres étudiants que j'avais, ils n'avaient de contrat avec personne!

(Professeur des universités, littérature).

Nous notons, d'autre part, que les injonctions à raccourcir la durée des thèses et à augmenter la part de thèses financées dans les laboratoires, ancrant la formation doctorale dans un format contractuel, sont plus massivement incorporées par les enseignants-

\footnotetext{
13 http://www.enseignementsup-recherche.gouv.fr/cid22130/les-cifre.html\#\#repartitiondomaine.

${ }^{14}$ L'AERES émet des recommandations systématiques sur ce point.

${ }^{15}$ Des auteurs montrent également que les formes d'investissement entrepreneuriaux sont variables, et que l'engagement académique des chercheurs ne disparaît pas nécessairement, même lorsque ceux-ci ont créé des entreprises (Lamy et Shinn, 2006). Dans notre cas, plusieurs rapports de l'AERES recommandent, dans les disciplines SHS les plus proches de la commande publique, de ne pas multiplier les contrats « alimentaires » et d'arrimer les recherches conduites à des questions de fond.

${ }^{16} \mathrm{http}: / /$ media.education.gouv.fr/file/10/8/7108.pdf.
} 
chercheurs en géographie, qui font trois fois plus mention de ce type de recommandation qu'en littérature, dans notre échantillon de rapports AERES. Les considérations relatives à l'insertion professionnelle des docteurs sont plus de deux fois plus présentes dans les rapports en géographie que dans ceux en littérature.

Les disciplines se positionnent alors différemment sur l'axe académique - professionnel hors académie, ce qui a des incidences sur le statut du doctorant. Comme le suggérait Louvel (2006), lorsque le pôle académique prédomine, les doctorants sont plus volontiers considérés comme des « futurs collègues » plutôt que des « jeunes collègues », contrairement à leurs homologues qui travaillent dans le cadre d'une relation contractuelle avec un employeur. Le fait que seuls environ $30 \%^{17}$ des thésards en lettres, parfois moins selon les universités, soient financés pour la réalisation de leur doctorat, ne favorise pas la reconnaissance de leur statut de travailleur et, par conséquent, leur possibilité d'émettre des revendications salariales. Plus encore, dans une proportion similaire, nombre d'entre eux réalisent leur thèse en étant salariés pour une autre activité principale. Ces doctorants font alors figure de bénévoles de la recherche : la thèse acquiert le statut d'activité secondaire voire de « hobby », bien qu'elle soit chronophage et engage son auteur à fournir un travail important.

Enfin, dans les rapports AERES, nous ne trouvons que deux occurrences, et uniquement en géographie, de recommandations visant à faire participer les doctorants aux décisions du centre, c'est-à-dire, à impliquer statutairement ce "personnel » dans la conduite et l'organisation du travail collectif, au même titre que les chercheurs et les administratifs.

Pour faire face à ces conditions précaires d'existence et, par conséquent, à l'invisibilisation de leur place dans le monde scientifique, des collectifs de jeunes chercheurs se sont constitués dans les années 2000. Ces mobilisations, souvent relayées par les enseignants-chercheurs, rendent visible la spécificité persistante de la condition doctorale. Celle-ci est caractérisée par une ambivalence instituée : le doctorant est l'un des rares personnels à pouvoir disposer, dans le même temps, d'une carte professionnelle, s'il donne des cours à l'université par exemple, et d'une carte d'étudiant. Il oscille alors, d'une part, entre un statut d'apprenti-professionnel, qui l'autorise à revendiquer dans le cadre d'une relation contractuelle l'obtention de ressources pour réaliser son travail, et voit son directeur de thèse tenu à son égard par une série d'obligations, d'autre part, un statut d'étudiant, qui certes le dégage des contraintes de la vie collective et l'affranchit de commandes exogènes, mais lui interdit du même coup de réclamer des droits et

\footnotetext{
17 Les chiffres nationaux sur le financement doctoral ont été compilés sur un blog: http://blog.educpros.fr/guillaume-miquelard-et-paul-francois/2015/12/08/quelqueschiffres-sur-le-doctorat-et-les-docteurs/, qui montre que $90 \%$ des thèses en sciences disposent d'un financement propre, tandis que ce nombre tombe à $33 \%$ en lettres et sciences humaines.
} 
une place ${ }^{18}$ dans des collectifs de recherche - dans la mesure où, dans les cas que nous avons soulevés, il travaille sans supérieur hiérarchique stricto sensu, ni rémunération dédiée.

Ainsi, la proximité ou l'éloignement de la (sous-)discipline au marché, la tendance des chercheurs à incorporer ou à tenir à distance des injonctions émanant des pouvoirs publics, peuvent affecter le positionnement des doctorants dans les laboratoires et le spectre de leurs débouchés professionnels. Mais les «cultures épistémiques » (KnorrCetina, 1999) des disciplines influent également sur la reconnaissance du travail du doctorant et sur la forme de son intégration professionnelle dans des collectifs de travail.

\subsection{Modalités de production du savoir et statut des doctorants}

Selon notre enquête, trois dimensions articulées sont enfin porteuses d'effets sur l'activité des doctorants et les formes de leur intégration: les conditions matérielles de la pratique de recherche, les modalités d'acquisition des compétences scientifiques, et, enfin, le degré de division (et de complémentarité) du travail.

Les conditions matérielles d'exercice de la pratique scientifique varient selon les disciplines. Le lieu de travail, le laboratoire, désigne souvent un espace configuré autour de la " paillass $^{19}$ » et d'autres instrumentations spécifiques nécessaires à la production du travail scientifique. Sous l'influence des sciences expérimentales, le terme de « laboratoire » a été importé en SHS. Or, dans plusieurs domaines, la pratique de recherche ne s'est pas construite autour d'équipements technologiques. Comme le souligne un enquêté :

Je connaissais un prof d'italien à Aix, qui était spécialiste de la correspondance d'un auteur italien du XVle siècle, il avait la moitié de la correspondance de cet auteur chez lui, soit en pièce originale soit en photocopie, donc il travaillait chez lui, ses doctorants, eh bien ils allaient le voir chez lui. Si vous voulez c'est pas pareil... Je ne suis pas sûr que ce soit bien, mais bon vous ne changez pas les pratiques... Ils n'ont pas de bureau, la majorité en sciences humaines. (Directeur de la section des laboratoires, AERES, Sciences expérimentales)

Weinberg (1970) observait une corrélation entre la sophistication des équipements et la formalisation des collectifs de travail. Les pratiques scientifiques et les instruments sur lesquels elles reposent conditionnent ainsi les formes de socialisation professionnelle. Or, bien que les injonctions réformatrices de la part des instances dirigeantes de

\footnotetext{
${ }^{18}$ Au sens matériel également : cette ambiguïté peut être maintenue par les centres de recherche étant donnée leurs faibles dotations en locaux, notamment en SHS. Le contexte de rareté budgétaire est déterminant ici.

${ }^{19}$ C'est ainsi que chercheurs en biologie désignent leur plan de travail, dont l'équipement varie selon les types de manipulations (« manip ») qu'ils pratiquent. En SHS, l'équivalent serait la table de bureau, lorsque les moyens en locaux le permettent.
} 
la profession en faveur d'une intégration plus collective du travail commencent à être suivies d'effets, la dimension individuelle des pratiques scientifiques continue de singulariser certaines disciplines des SHS. Le cas que nous prenons pour objet d'étude contraste ainsi avec ceux que la littérature a renseignés. Shinn observait, en physique, une complémentarité des rôles et des missions à l'intérieur des laboratoires (Shinn, 1988). Les « juniors », c'est-à-dire les doctorants et post-doctorants, font partie d'un tout. Ils développent des compétences spécialisées, et sont appelés à produire des résultats d'un certain type, qui s'intègrent dans le processus plus global de production des connaissances. L'organisation de la recherche sur projets, lorsqu'elle est dominante, favorise considérablement cette forme de division du travail. Or ce modèle d'intégration quasi organique des travaux des membres de différents statuts se rencontre plus rarement en SHS, en particulier en sciences humaines. En effet, les sujets de thèses ne sont pas nécessairement arrimés à un projet de recherche collectif, alors que c'est souvent le cas en biologie ou en nanotechnologie (Barrier, 2011), par exemple. Les travaux des doctorants ne s'ancrent pas toujours dans le développement d'un programme de laboratoire, ce qui induit une interdépendance plus discrète de leurs activités vis-à-vis de celles des « seniors ». Ainsi, une dimension centrale de la professionnalisation, l'acquisition d'une place dans un collectif, est plus ou moins favorisée par l'organisation du travail scientifique.

Enfin, si le travail doctoral et les relations entre les doctorants et leurs directeurs se sont considérablement formalisés depuis les années 1990 (Serre, 2015), les dimensions matérielles et organisationnelles de la production de la science induisent des relations pédagogiques à géométries variables entre le doctorant en SHS et son encadrant, tout comme le sont les formes d'apprentissage des savoirs et des savoir-faire. D'autres enquêtes qualitatives approfondies permettraient de documenter plus avant ces relations. Cependant, nous pouvons souligner que la proximité entre l'encadrant et le doctorant est d'autant plus forte lorsque la formation à la discipline suppose une maitrise des procédés techniques et des manipulations potentiellement dangereuses, comme le souligne Louvel en prenant le cas de la biologie (Louvel, 2006). Dans certaines disciplines des sciences humaines, la formation du doctorant passe plus rarement par la transmission de ces types de savoir-faire qui rendent nécessaire un échange soutenu entre les postulants et les titulaires. Cette propension se combine avec des variations démographiques importantes : le nombre de doctorants par encadrant en SHS est plus élevé en moyenne que ceux des autres domaines (AERES, 2007), rendant plus délicats un suivi constant des travaux des jeunes chercheurs, mais aussi leur intégration dans des projets collectifs du laboratoire.

Les modalités de la formation du doctorant en SHS au travail académique sont hétéroclites. Elles varient selon les disciplines considérées, au gré de l'introduction plus ou moins prégnante des réformes indirectes de l'organisation de la recherche (via la prolifération des financements sur projets) dans les différents domaines académiques, 
mais également en fonction de l'évolution des pratiques mêmes de recherche (développement d'outils collaboratifs, de méthodes et d'objets d'investigation requérant un travail collectif et/ou une spécialisation des tâches), qui se diffusent, avec une ampleur variable, dans les sciences humaines et sociales.

\section{Conclusion}

Les doctorants ou les jeunes docteurs ont été intégrés, investis profondément dans l'exposition de la recherche, la publication de la recherche, la conception de la recherche. (Professeur des universités, littérature)

[Aujourd'hui], les jeunes chercheurs sont bien mieux formés à la recherche que ce qu'on était. En réalité, [...] on n'était pas formé à la recherche ! (Professeur des

universités, géographie).

Nous avons choisi d'aborder l'évolution du rôle et de la place du doctorant « de biais », telle qu'elle se donne à lire dans les discours et les réformes entreprises par les acteurs de la régulation des politiques scientifiques, pour ensuite proposer des clés de compréhension des formes variables de leur professionnalisation. Notre approche de ces évolutions complète les analyses empiriques du vécu des doctorants eux-mêmes et de leur rapport aux mondes professionnels dans lesquels ils s'intègrent, pour explorer les facteurs, moins fréquemment abordés, de l'hétérogénéité des formes prises par leur professionnalisation.

En déplaçant la focale, nous avons montré que l'évolution des formes de socialisation des doctorants voit en partie le jour à travers la posture réformatrice de membres d'instances normatives, qui ont joué un rôle actif dans la construction d'une « nécessité » à faire du doctorant un personnel à part entière des centres de recherche, qui s'y insère et s'acculture ainsi aux règles du monde scientifique et/ ou non-académique. Cette réflexion permettait alors d'aborder des effets d'isomorphisme, certaines disciplines des SHS étant encouragées à se structurer sur des modèles empruntés aux sciences expérimentales - fonctionnement « organique » des laboratoires, caractérisés par une interdépendance entre des membres de différents statuts. En filigrane, c'est la nécessité de réformer les méthodes de travail et d'organisation scientifique du travail en SHS qui se donne à lire dans les discours réformateurs étudiés. Le questionnement peut alors être renversé : alors que seuls quelques travaux de sociologie de la profession académique se centrent sur les doctorants, analyser la place et le rôle qui leur sont dévolus met en lumière les formes de l'organisation scientifique actuelle.

C'est pourquoi nous avons ensuite distingué les conditions sociales qui permettent à ces injonctions de se traduire dans les pratiques. L'ouverture des disciplines au marché et à la société civile, le degré de division du travail et les modes de production du savoir qui les caractérisent, sont des facteurs - eux-mêmes dynamiques - qui influent sur la place du doctorant voire sur la nature de ses activités. Notre étude prolonge ainsi les 
travaux qui mettent en lumière les logiques de différenciation interne aux groupes professionnels (Demaziere et al., 2009) : la pluralité des formes même d'apprentissage et de socialisation au métier montre que derrière la dénomination couramment employée de "communauté scientifique», celle-ci se compose plutôt de mondes professionnels distincts.

Le choix d'étudier les doctorants en SHS complète alors les résultats de la littérature portant sur d'autres domaines, en mettant en évidence la spécificité des formes de leur professionnalisation. Alors que Frances (2012) concluait à la transformation du doctorant de biologie en entrepreneur, ce résultat n'est pas généralisable aux différentes disciplines. Malgré de fortes incitations à conduire des recherches partenariales et ouvertes au monde de l'entreprise, y compris en SHS, et bien que nous observions une diversification des régimes de professionnalisation des doctorants, c'est d'abord au développement d'une professionnalisation au travail académique que nous assistons - notamment en sciences humaines. Si ces apprentis-professionnels développent des compétences qui les prédisposent à entrer sur le marché scientifique, une part d'entre eux parvient cependant à les valoriser dans d'autres secteurs professionnels, souvent pour faire face au faible nombre de postes d'enseignants-chercheurs au regard du nombre de thèses soutenues chaque année.

\section{Bibliographie}

BARRIER J. (2011), «La science en projets : financements sur projet, autonomie professionnelle et transformations du travail des chercheurs académiques », Sociologie du travail, vol. 53, p. 515-536.

Chao M., Monini C., Munck S., Thomas S., Rochot J, Van de Velde C. (2015), « Les expériences de la solitude en doctorat. Fondements et inégalités», Socio-logos, vol.10, consulté le 9 décembre 2016. En ligne. URL : http://socio-logos.revues.org/2929.

DEMAZIERE D. (2009), « Professionnalisations problématiques et problématiques de la professionnalisation », Formation emploi, vol. 108, p. 83-90.

DemaZiere D., GADÉA C. (dir.) (2009), Sociologie des groupes professionnels, Paris, La Découverte.

EsPEland, W. (2015), «The History of 10 \%: Social Science Measures and the Construction of Gay Identities », Communication at the CSO's scientific seminary, Centre de sociologie des organisations, CNRS/Sciences Po, 25 avril 2015.

FRANCES J. (2012), « Portrait du doctorant en entrepreneur. Les Doctoriales : un outil "au service de la professionnalisation des doctorants” ?", Mouvements, vol. 3, nº 71 , p. 54-65.

FREIDSON E. (1984), La profession médicale, Paris, Payot. 
GozLAN C. (2016), « Les Sciences Humaines et Sociales face aux standards d'évaluation de la qualité académique. Enquête sur les pratiques de jugement dans une agence française », Sociologie, vol. 7, n³, p. 261-280.

KNORR-CETINA K. (1999), Epistemic cultures. How the sciences make knowledge, Cambridge, Harvard University Press.

LAMY E., SHINN T. (2006), « L'autonomie scientifique face à la mercantilisation. Formes d'engagement entrepreneurial des chercheurs en France ", Actes de la recherche en sciences sociales, vol. $164, \mathrm{n}^{\circ} 4$, p. 23-50.

LOUVEL S. (2006), «Les doctorants en sciences expérimentales: futurs collègues ou jeunes collègues ? », Formation emploi, vol. 96, p. 53-66.

SERRE D. (2015), « Être doctorant-e. Socialisations, contextes, trajectoires », Socio-logos, vol.10, consulté le 9 décembre 2016. En ligne. URL: http://socio-logos.revues.org/2924.

SHINN T. (1988), "Hiérarchies des chercheurs et formes de recherche», Actes de la recherche en sciences sociales, vol. 74, p. 2-22.

VILTER S. (2008), « Doctorants et docteurs de l'UVSQ : les conditions de réalisation de la thèse », Université de Versailles Saint-Quentin en Yvelines - Observatoire de la Vie Étudiante, p. 1-8.

Weinberg A. (1970), « Scientific Teams and Scientific Laboratoires », Daedalus, vol. 99, p. 1056-1975. 